\title{
Maternidad subrogada: análisis de los derechos fundamentales
}

\author{
Yuly Luz Motta Angulo \\ XXX@ucv.edu.pe \\ luz_motta@hotmail.com \\ Universidad Cesar Vallejo. \\ Tarapoto, Perú
}

\section{RESUMEN}

El objetivo del estudio nace con la necesidad de identificar los principales hallazgos sobre la maternidad subrogada, en base a un análisis de los derechos fundamentales, por lo cual la investigación se orientó a una metodología descriptiva, por lo cual se aplicó como instrumento una ficha de análisis documental o bibliográfica a los 20 artículos científicos seleccionados como unidad de análisis. Entre los hallazgos más significativos se pudo conocer que esta práctica no está aceptada ni regulada por los sistemas jurídicos de la mayor parte de países debido a que vulnera de forma directa algunos de los derechos que le corresponden al menor y a la madre biológica, y a pesar de que legitima los derechos de los padres intencionales, existiendo de esta forma una controversia o conflicto jurídico entre las partes implicadas, presentándose adicionalmente a ello otros conflictos éticos y morales. En función a ello, resulta necesario que los países dispongan de normativas orientadas a regular la maternidad subrogada, considerando la realidad que presenta cada país.

Palabras clave: derecho a la identidad; derechos fundamentales; maternidad subrogada. 


\title{
Surrogacy: analysis of fundamental rights
}

\begin{abstract}
The objective of the study was born with the need to identify the main findings on surrogacy, based on an analysis of fundamental rights, for which the research was oriented to a descriptive methodology, for which an instrument of Documentary or bibliographic analysis of the 20 scientific articles selected as the unit of analysis. Among the most significant results it was possible to know that this practice is not accepted or regulated by the legal systems of most countries because it directly violates some of the rights that correspond to the minor and the biological mother, and despite that legitimizes the rights of the intentional parents, thus existing a controversy or legal conflict between the parties involved, additionally presenting other ethical and moral conflicts. Based on this, it is necessary for countries to have regulations aimed at regulating surrogacy, considering the reality that each country presents.
\end{abstract}

Keywords: right to identity, fundamental rights, surrogacy.

Artículo recibido: 10 Agosto. 2021 Aceptado para publicación: 07. Setiembre. 2021

Correspondencia: luz_motta@hotmail.com Conflictos de Interés: Ninguna que declarar 


\section{INTRODUCCIÓN}

En la actualidad, la constante evolución de la medicina humana ha suscitado que se aprueben una serie de técnicas asociadas con la reproducción asistida, siendo esta considerada como una alternativa de solución frente a problemas de infertilidad, por lo cual la maternidad subrogada representa una acción generosa por medio de la cual una persona da su consentimiento para ser portadora del embarazo mediante la implantación o inseminación del embrión concebido. No obstante, esta práctica no suele ser aceptada en diversos sistemas jurídicos pues se presume de una vulneración de los derechos fundamentales del embrión, madre sustituta y progenitores, principalmente el derecho a la identidad del embrión, debido a que existe una manipulación consentida del mismo por parte de los padres. Cabe precisar además que numerosos países no tienen a su disposición una normativa reguladora de la maternidad subrogada, lo cual ocasión que se presente numerosos inconvenientes durante el nacimiento y reconocimiento del niño

Es así como, se presume la vulneración de los derechos fundamentales de los recién nacidos a través de la maternidad subrogada debido a que suscitan diversos inconvenientes jurídicos que afectan el derecho de identidad, dignidad, filiación, a la protección de la familia, entre otros derechos que impiden que esta práctica pueda ser permitida y regulada por la legislación de la mayoría de los países.

En lo que refiere a los avances teóricos, la maternidad subrogada según Torres, et ál. (2019) es una técnica de reproducción asistida poco tradicional en la cual una mujer presta su vientre ya sea mediante un contrato remunerado o por solidaridad para gestar el bebé de una pareja que no ha podido tener hijos, pero sí tener derecho alguno sobre el recién nacido. Por su parte Vasilieva, et ál. (2018), alude que la maternidad subrogada consiste en la intervención de una madre sustituta que tiene como propósito gestar al bebé de una pareja que por medios naturales o a través de tratamiento no ha podido tener hijos. Asimismo, Cossutta (2018), alude que la gestación o maternidad subrogada es aquella práctica en la cual una mujer lleva a cabo todo el proceso gestacional y da a luz a un bebé que genéticamente pertenece a otros padres. También, en relación con sus características, Ales (2020), la maternidad subrogada se caracteriza por ser un tratamiento altruista que permite mitigar o hacer frente a la infertilidad que padecen muchas parejas por distintos motivos. 
Por otro lado, para Gonzáles (2019), los derechos fundamentales son aquellos atributos incluidos o vinculados a la constitución o carta de derechos de cada país, que tienen como finalidad garantizar el respeto por la dignidad de una persona. En tanto, para Múrtula (2020) son aquellos privilegios o garantías inherentes a cualquier persona, los mismos que se encuentran dogmatizados en el ordenamiento jurídico de un determinado país. Del mismo modo, Valero (2019) puntualiza que los derechos fundamentales son considerados como atributos que posee toda persona en la cual la constitución u ordenamiento jurídico de un determinado país garantiza el respeto sobre la dignidad de la misma. En tal sentido, es importante destacar al derecho a la identidad y la verdad, la cual es considerada como un privilegio fundamental que tiene todo individuo desde su concepción, el cual implica el derecho a una identidad psicológica, social, histórica y sobre todo a una verdad biológica, garantizando de este modo el conocimiento sobre la verdad de su procedencia y origen parental, el mismo que es establecido y reconocido mediante el proceso de filiación. (Posadas, 2018)

Así también, respecto a los antecedentes investigativos internacionales, Sarasol, C. y Ramón, F. (2021), La gestación subrogada: Aspectos éticos y jurídicos en el derecho español; concluyeron que la maternidad subrogada no está legalmente permitida en España, sin embargo, aquellas familias con un rango económico acceden a dicha práctica bajo la modalidad de ilegalidad aprovechándose de aquellas mujeres cuyas condiciones de vida son escasas, siendo considerado además como un acto ilícito que vulnera los derechos fundamentales del bebé nacido como por ejemplo el derecho a la identidad, el derecho a la dignidad propia de la persona, el derecho a la verdad, el derecho a tener decencia, derecho al propio cuerpo y el interés superior del menor. Igualmente, BarahonaCobos, D. y Guerra-Coronel, M. (2021), La maternidad subrogada en el Ecuador; concluyeron que desde el año 2008 la constitución del Ecuador confiere un mayor compromiso a los derechos fundamentales sobre cualquier disposición constitucional o mandato legal, donde la Carta Magna sacraliza entre los derechos fundamentales de una persona; el derecho a beneficiarse y acceder a los avances científicos y tecnológicos, al igual que el derecho a elegir de forma libre a los métodos o alternativas de salud sexual y reproductiva, e incluso decidir por su propia voluntad el número de hijos que desea concebir y es por ello que el Ordenamiento Jurídico de Ecuador tiene como principal propósito garantizar el respeto por el derecho a gozar de los avances médicos de 
reproducción siempre y cuando no se vulneren los derechos fundamentales. También, Valero, A. (2019), La maternidad subrogada: Un asunto de derechos fundamentales; concluyó que en España no está autorizada ni mucho menos legalizada la práctica de la maternidad subrogada, por cuanto no siempre resulta del todo favorable pues esta maternidad se ejerce a través de un contrato remunerado, donde las madres sustitutas en algunos casos terminan negándose a entregar al recién nacido aludiendo que tienen los mismos derechos que el mismo, produciendo así serios inconvenientes legales que la Jurisprudencia del Tribunal Europeo de Derechos Humanos, quien determina que los derechos como madre se acreditan a la persona que llevó todo el proceso gestacional pese a no compartir la misma genética. Del mismo modo, Arroyo, A. (2020), Gestación por sustitución: La dignidad humana en juego; concluyó que desde la Jurisdicción de España, en conjunto con el Tribunal Europeo de Derechos Humanos, la maternidad subrogada es considerada como una práctica de reproducción asistida que en la actualidad no es permitida ni mucho menos legalizada, puesto que va en contra y vulnera los derechos fundamentales del recién nacido, poniendo de este modo en juego la dignidad humana y la vulneración del derecho a la identidad, a la verdad y el interés propio del niño. Asimismo, Martínez-Martínez, V. (2015), Maternidad subrogada. Una mirada a su regulación en México; concluyó que la maternidad subrogada se encuentra regulada por las normativas mexicanas de forma parca y disímil, cuyas normativas sólo pueden ser aplicadas en ciertas áreas geográficas del país, mientras que en otras áreas se evidencia un vacío normativo referente a esta técnica debido a que afecta en gran medida los derechos fundamentales, principalmente el derecho a la libertad; cabe mencionar también que para su regulación es sumamente importante tener en cuenta una serie de factores asociados de acuerdo a su naturaleza, cualidades y rasgos específicos, teniendo gran cuidado en el interés superior del niño, derechos de la mujer e inherentes al individuo tales como la dignidad e intimidad. Por su lado, Lamm, E. (2016), Una vez más sobre gestación por sustitución, porque sin marco legal se siguen sumando violaciones a derechos humanos; concluyó que la ausencia de una regulación pertinente sobre la maternidad subrogada ocasiona que los derechos sean vulnerados o violados en gran medida, por tanto, se reconoce la necesidad de que el Estado promueva la implantación de un marco jurídico direccionado a privilegiar y representar una garantía para el libre ejercicio de los derechos, respetando y promoviendo el derecho de los individuos a gozar 
de una paternidad y maternidad responsable y libre, permitiendo reconocer además la diversidad de personas que integran la sociedad para volverla más diversa, plural e incluyente. De igual forma, Castellanos, J. (2019), Análisis de la maternidad subrogada como nueva tecnología en el ámbito biomédico y jurídico-filosófico. Avance técnico, retroceso humano; concluyó que el análisis minucioso y detallado de las normas y jurisprudencias respecto a los supuestos que se produjeron y consideraron en la realidad implica que se garanticen los derechos de los progenitores intencionales, así como de la gestante y el menor; por tal motivo, la dignidad de la progenitora biológica y el menor evita que se lleve a cabo la maternidad subrogada, por cuanto deducen que esta práctica sólo busca satisfacer los supuestos de derecho de paternidad de los individuos, el cual no tiene consistencia jurídica y vulnera la dignidad de las partes mencionadas con anterioridad. Así también, Fernández, P. (2018), Gestación subrogada, ¿cuestión de derechos?; concluyó que los inconvenientes vinculados con la cuestión y variedad del enfoque del gobierno ocasiona que no se puedan efectuar avances significativos al respecto, por lo que resulta poco conveniente aprobar una norma que regule de forma mínima esta figura con un alcance internacional, sin desistir en la propuesta; asimismo, informes previos alertaban de amenazas considerables sobre los derechos de las personas, esencialmente de los niños, respecto a los contratos de esta figura donde destacan las mencionadas a continuación: abandono del menor, inadecuación de los comitentes para asumir la responsabilidad de ser progenitores, derecho del menor a conocer a sus progenitores biológicos, malas prácticas durante la maternidad subrogada, entre otros. Además, Lázaro, C. (2019), El concepto de persona como elemento clave de la identidad europea: El caso de la maternidad subrogada; concluyó que el derecho a la identidad, como parte de la vida privada, es uno de los derechos fundamentales que se ve afectado en mayor medida por esta nueva figura; por lo que, en base a tal premisa, el Art. 8 del CEDH tipifica el respeto a la vida privada donde todos los individuos deben establecer los detalles de su identidad como seres humanos, incluyendo también su filiación, donde argumenta también que la definición de la vida privada a la filiación se manifiesta cuando existe un vínculo directo entre el menor o menores nacidos como producto de la maternidad subrogada y establecimiento jurídico de la filiación. De igual manera, Matia, F. (2019), ¿Resulta oportuno dar un tratamiento jurídico a la gestación subrogada en nuestro país?; concluyó que el tratamiento jurídico es indispensable frente a la 
maternidad subrogada teniendo en consideración ciertas precisiones previas tales como la delimitación sobre la retribución y el altruismo, donde se asume que los progenitores intencionales son los responsables de asumir los gastos que derivan del cuidado del futuro niño y demás perjuicios económicos que se ocasionen en la embarazada; también, se debe analizar si esta gestación puede ser de libre voluntad entre las partes implicadas o su viabilidad sería condicionada con diferentes circunstancias; además, se debe avalar por la libertad de las partes implicadas con el propósito de soslayar abuso de cualquiera de las partes; de igual modo, se debe asegurar los derechos de las partes implicadas y principalmente el interés superior del menor. En tanto, Vilar, S. (2019), La inseguridad jurídica derivada de la insuficiente regulación de la gestación subrogada en España; concluyó que la regulación de esta figura es necesaria dentro de la realidad que se percibe por cuanto contribuiría con la reducción de los problemas sociales y garantiza el respeto de los derechos de las partes involucradas, principalmente del futuro niño, por lo que es fundamental que se establezca de modo detallado las limitaciones y requisitos que deben ser cumplidos en su totalidad, considerando en todo momento que el interés superior del niño debe ser priorizado en cada uno de los procesos, así como la necesidad de tener un consentimiento informado de las partes implicadas. Del mismo modo, Ávila, C. (2017), La maternidad subrogada en el Derecho Comparado; concluyó que tras el análisis de la legislación internacional permitió reconocer que la práctica de la maternidad subrogada está prohibida dentro de Francia, Alemania, Suiza, Italia y Austria, por cuanto esta práctica vulnera en gran medida los derechos fundamentales de la madre biológica y menor principalmente; además, se evidencia que Reino Unido, Canadá, Grecia, Brasil e Israel, esta práctica es aceptada siempre y cuando exista un propósito altruista, disponiendo de esta forma los procesos que deben seguirse de forma debida para dar inicio al tratamiento respectivo; igualmente, entre los países que admiten de forma amplia esta práctica destaca Rusia, Ucrania, e India; mientras que Australia, México y Estados Unidos son aquellos países donde esta práctica se encuentra regulada dentro de su sistema federal. Así pues, Emaldi, A. (2018), La maternidad subrogada vulnera el principio constitucional de la seguridad jurídica. La imperiosa necesidad de buscar una solución al problema español: cambio legislativo o cumplimiento de la ley; concluyó que el principal argumento en contra de la maternidad subrogada fue el riesgo que ello implica respecto a la vulneración de sus derechos fundamentales, para lo cual es necesario 
también que se reflexione sobre la probabilidad del derecho del menor sobre conocer su origen biológico; en cambio, el principal argumento a favor de esta práctica es que ello contribuiría con el cumplimiento del derecho a la libertad reproductiva de las progenitores intencionales, por cuanto ello podría respaldarse en la libertad para el desarrollo de la personalidad y dignidad de las personas. Por otro lado, Garibo, A. (2017), El interés superior del menor en los supuestos de maternidad subrogada; concluyó que el análisis detallado de la regulación respecto a la figura estudiada reconoció que la progenitora subrogada cuenta con el respaldo normativo para evitar que se encuentre en una situación de explotación, donde la opción más favorable es que el contrato no se caracterice por ser oneroso; sin embargo, por otra parte, el menor subrogado no cuenta con el respaldo jurídico suficiente para que no sea afectado por la separación de su madre biológica, evitando además que sufra problemas psicosociales o de identidad debido a la dificultad para conocer su origen biológico y demás problemas que vulneraría el interés superior del niño. Al mismo tiempo, Martínez, H. (2018), Maternidad subrogada; concluyó que la modalidad de la maternidad subrogada transgrede una serie de principios jurídicos, principalmente el interés superior del niño, lo cual permite reconocer además que la sola acción de requerir una serie de elementos e intervención de diversos sujetos, es una figura normativa que debe desarrollarse en una perspectiva normativa; además, se evidencia que los contratos de esta modalidad fomenta el interés lucrativo y desvirtúa la esencia de la maternidad, afectando de esta forma la dignidad de la mujer. Ahora bien, Romero, C. (2018), Maternidad subrogada: lagunas en el ordenamiento jurídico colombiano. ¿Con qué elementos cuenta el juez para adoptar su decisión?; concluyó que la maternidad subrogada no se encuentra impedida por el orden jurídico colombiano pero tampoco cuenta con una relación concreta, por lo que el juez tiene la potestad para determinar de forma autónoma, discreta y legal una decisión razonable teniendo en cuenta los principios generales del derecho orientados a regular el proceso y velar por el respeto de los derechos de las partes implicadas respecto a la igualdad, libertad en el desarrollo de personalidad y dignidad para la madre biológica, y garantizar la prevalencia del interés superior del niño a favor del menor. Finalmente, Regalado, M. (2016), Efectos, consecuencias y regulación de la maternidad subrogada; concluyó que las doctrinas nacionales e internacionales concernientes a la materia de estudio permiten reconocer la necesidad de implantar una posición clara y precisa respecto a la prohibición de su regulación por 
cuanto existen una serie de dificultades que ello abarca, siendo una de las principales la protección de los derechos de las personas implicadas que, pese a no contar con un respaldo constitucional efectivo, exigen que se brinde una protección jurídica en base a ciertos principios, garantías y valores respectivos.

Por otro lado, referente a los antecedentes investigativos nacionales, Rupay, L. (2018), La maternidad subrogada gestacional altruista en el Perú: Problemática y desafíos actuales; concluyó que la maternidad subrogada no está permitida ni legalizada en el Perú, pues las únicas prácticas de reproducción asistida que son permitidas son donde la madre gestante y la madre progenitora recaiga en la misma persona, razón por la cual muchas parejas que no ha podido procrear de forma natural o través de tratamientos o prácticas de reproducción asistida permitidas y generalmente se opta por realizarlas en países extranjeros, sin embargo, no se consideran los inconvenientes legales presentados al tratar de ingresar a los hijos al Perú, pues la Constitución no permite que un recién nacido procreado bajo la modalidad de maternidad subrogada sea registrado bajo tutela de los progenitores pues se considera como madre a aquella persona que llevó el proceso gestacional, quedando demostrado además que la maternidad subrogada vulnera el libre desarrollo de la personalidad, la autonomía reproductiva y la protección familiar. Por su parte, Fiestas, R. y Chanduví, V. (2017), La maternidad subrogada y la impugnación judicial de paternidad en el Perú; concluyeron que la principal causa del impedimento para la aceptación de la paternidad en los casos de maternidad subrogado en el Perú es debido a la deficiente regulación jurídica que existe con respecto a las prácticas de reproducción asistida, cabe señalar que a nivel internacional esta práctica no es permitida ni legalizada puesto que según el Tribunal Europeo de Derechos Humanos la maternidad subrogada es una práctica que va en contra de los derechos fundamentales del niño, en ese sentido muchos padres subrogantes recurren a la vía judicial con la finalidad de quedarse y registrar a un recién nacido que biológicamente no les pertenece. Del mismo modo, Núñez, A. (2015), Derechos reproductivos de la mujer infértil en el Perú: acceso a la técnica de reproducción asistida de maternidad subrogada; concluyó que, desde otra perspectiva, esta técnica representa uno de los derechos fundamentales concernientes a los derechos reproductivos en función a 3 criterios relevantes de no discriminación, igualdad y autonomía, por lo que debe ser permitido que todas las mujeres infértiles puedan emplear la maternidad subrogada como mecanismo orientado a la consecución 
del proyecto de vida de la misma; no obstante, de ello derivan una serie de conflictos que abarcan una regulación de las políticas de salud, accesibilidad a esta técnica y caracterización de las personas que podrían acceder a esta técnica.

En tal sentido, frente a la realidad evidenciada en diversos contextos, se consideró conveniente proponer como objetivo: Identificar los principales hallazgos sobre la maternidad subrogada, en base a un análisis de los derechos fundamentales.

\section{MÉTODO}

Para desarrollar la investigación fue necesario emplear un diseño descriptivo, porque se pretende conocer de forma detallada la realidad que se percibe en diversos contextos respecto a la maternidad subrogada de tal manera que se pueda describir los principales hallazgos alcanzados durante los últimos años en base al análisis de los derechos fundamentales (Arbaiza, 2019).

De igual modo, para la recolección de datos e información se empleó como instrumento una ficha de análisis documental o bibliográfica puesto que se llevará a cabo un proceso reflexivo y analítico en base a la información presentada por diversos investigadores que hacen referencia sobre las variables en estudio (Ortega-Carbajal, et ál., 2015).

Por otro lado, los participantes son unidades requeridas para el recojo de información por cuanto, en base a la información extraída de estos, se procede a desarrollar cada uno de los objetivos propuestos inicialmente en la investigación científica (Ventura-León y Barboza-Palomino, 2017). En efecto, los participantes estuvieron representados por 20 artículos científicos desarrollados en diferentes contextos, es decir, fueron seleccionados para ser objeto de estudio de tal manera que, en función a los resultados alcanzados se pudo dar a conocer los principales hallazgos correspondientes a la maternidad subrogada durante los últimos años en base a un análisis de los derechos fundamentales.

También, respecto a los procedimientos desarrollados, inicialmente se ha efectuado la búsqueda de investigaciones asociadas con el tema en cuestión, las mismas que fueron desarrolladas durante los últimos años en diferentes contextos con la finalidad de extraer la información requerida para proceder a identificar y analizar de forma minuciosa el contenido de los mismos sobre la maternidad subrogada de tal manera que estos puedan ser presentados en el apartado de resultados.

De igual modo, para el análisis de datos se ha empleado el método cualitativo al momento de llevar a cabo la lectura e interpretación de la información extraída de las diversas 
fuentes bibliográficas, permitiendo de esta forma identificar los hechos más significativos relacionados al objeto de estudio.

Finalmente, se reconoce que no existieron conflictos de intereses durante el desarrollo de la investigación, por cuanto el propósito principal de la investigadora fue identificar los hallazgos de mayor relevancia que se presentaron durante los últimos años respecto a la maternidad subrogada en función a un análisis de los derechos fundamentales. De igual manera, se tuvo en cuenta los principios éticos básicos de la investigación.

\section{RESULTADOS}

Investigaciones realizadas acerca de la maternidad subrogada permitió conocer la realidad que se presenta en diversos ámbitos respecto a la regulación, principios generales y procesos jurídicos que ello implica, a la cual se debe regir esta práctica con la finalidad de velar en todo momento por el respeto de los derechos fundamentales del menor, madre gestante y progenitores intencionales. Así pues, con el transcurso de los años, diversos países buscaron implementar procedimientos a seguir y criterios a aplicar para que esta práctica puede llevarse a cabo de forma apropiada pese a la existente cuestión y controversia que se presenta sobre una posible vulneración o legitimidad de los derechos fundamentales de las partes involucradas, de acuerdo con la perspectiva que presenta cada uno. Por ejemplo, desde una perspectiva del menor, se evidencia la vulneración de los derechos a la identidad y la verdad, porque la práctica de la maternidad subrogada impide que el menor pueda tener conocimiento real sobre su historia, procedencia u origen biológico; adicionalmente a ello, se reconoce una transgresión del derecho a la filiación debido a que el menor no puede reconocer ser hijo biológico de sus padres. Por otra parte, desde la perspectiva de la madre gestante, esta práctica afecta la dignidad e integridad física y moral dado puesto que coloca al cuerpo de la madre biológica, con sus implicaciones físicas, psicológicas, sentimentales, racionales, etc., como objeto de comercio; de igual modo, ocasiona una ruptura de la unidad de la persona al ser considerado y tratado como objeto de un contrato; por tal motivo, por medio del contrato se debe velar por la integridad y bienestar de la madre biológica frente a las diversas circunstancias que puedan presentarse durante el embarazo, las mismas que podrían ocasionar perjuicios o daños considerables a la misma. Por último, desde la perspectiva de los progenitores intencionales, tal practica representa el ejercicio del derecho de reproducción o libertad reproductiva para aquellas personas que no gozan de una vida 
reproductiva adecuada, favoreciendo de esta manera a que se cumpla sus anhelos de tener una familia, reconociendo también el principio a la igualdad y no discriminación reproductiva y respetando así el derecho a tener una familia y a gozar de los diversos beneficios que proporcionan los avances científicos y tecnológicos; por tanto, a través del contrato se debe asegurar que tras el inicio de los procesos correspondientes o después del nacimiento del menor se va a realizar la entrega correspondiente del mismo, así como la respectiva inscripción del menor como hijo(s) de estos ante los organismos competentes.

De igual manera, respecto a los criterios internacionales que se deben tener en cuenta durante los procesos concernientes a la maternidad subrogada, se deduce que las autoridades y organismos competentes deben velar en todo momento por el respeto del interés superior del niño, por cuanto este principio es la base para el desarrollo de esta práctica mediante la cual se determina que todos los recién nacidos deben gozar de una convivencia óptima dentro de un grupo familiar, así como disponer del cuidado y la protección jurídica-constitucional necesaria con el propósito de que pueda crecer dentro de un entorno idóneo para desarrollar las habilidades y destrezas propias de su edad.

En efecto, la maternidad subrogada, desde un análisis de los derechos fundamentales, representa una práctica que en la actualidad representa una controversia debido a las diferentes posiciones que se toman en torno a ella donde la ausencia de una debida regulación en gran parte de los países objeto de estudio ha ocasionado una serie de irregularidades y conflictos legales durante el seguimiento de los procedimientos respectivos, atentando de esta forma el orden público y las buenas costumbres que deben ser legitimadas para ser debidamente ejercidas. Por tal motivo, para que la práctica pueda llevarse a cabo de forma conveniente, es importante que se priorice en todo momento el interés superior del niño, al ser este un principio esencial que vela por la integridad y bienestar del menor.

\section{DISCUSIONES}

Después de haber llevado a cabo un análisis sistemático detallado de los resultados expuestos por las diversas investigaciones desarrolladas en base a la realidad que se presenta en un ámbito internacional y nacional respecto a la maternidad subrogada, en base a un análisis de los derechos fundamentales, permitieron deducir que esta práctica 
abarca aspectos jurídicos, éticos y morales debido que, al no estar reguladas por la mayoría de los países, los progenitores intencionales buscan emplear mecanismos ilícitos que afectan en gran medida los derechos de las demás partes involucradas, especialmente al menor, visto que al ingresar a aquellos países en donde esta práctica no está permitida, los menores nacidos se involucran en procesos legales sumamente complejos, tal y como fundamenta Rupay (2018), donde varias parejas que presentan dificultades para concebir un hijo, acuden a lugares del exterior para llevar a cabo esta práctica, sin embargo, presentan serias complicaciones al no poder ingresar nuevamente a su país de origen donde la normatividad reguladora impide el registro de los menores que fueron provenientes de esta práctica dado que establece que, desde una perspectiva biológica, el menor no les pertenece y por ende no deben estar bajo su tutela.

Adicionalmente a ello, se evidencia también que la maternidad subrogada conlleva a la aparición de problemas en los menores que nacieron bajo esta práctica, donde Garibo (2017) sostiene que entre los problemas más significativos destaca los problemas psicosociales que podrían originar crisis familiares serias o disfunción dentro del hogar en el que se desarrollan, problemas de identidad al no tener conocimiento alguno sobre su origen y experimentar una sensación de incertidumbre sobre quienes son.

Es así como las sociedad y autoridades jurídicas presentan una serie de dificultades para garantizar que esta práctica se encuentre debidamente regulada por una norma o ley, velando de esta manera por la integridad y bienestar de los sujetos implicados. De acuerdo con ello, es preciso destacar a Lamm (2016), por cuanto manifiesta que la falta de normativas orientadas a regular la maternidad subrogada representa un mayor riesgo de vulneración de los derechos de los sujetos involucrados, siendo necesario para ello que el gobierno incentive la creación de una nueva normativa acorde con la realidad percibida. Bajo ese enfoque, es propicio hacer énfasis en lo sustentado por Núñez (2015), quien menciona que esta esta práctica contribuiría con el cumplimiento de los derechos fundamentales basados en 3 criterios determinantes de igualdad, no discriminación y autonomía, por lo cual debe respetarse el derecho de las personas que deseen convertirse en padres y formar una familia, siendo indispensable para ello tener en consideración las legislaciones, criterios, principios, leyes, etc., internacionales y nacionales.

\section{CONCLUSIONES}


La revisión sistemática de las investigaciones relacionadas con el tema en cuestión permitió reconocer que la maternidad subrogada es una de las prácticas poco usuales que se encuentran reguladas por una cantidad mínima de los sistemas jurídicos de los países estudiados alrededor del mundo. En tal sentido, se deduce que existe una evidente vulneración de los derechos fundamentales del menor por cuanto transgrede su derecho a la identidad y a la verdad, al no tener conocimiento sobre su verdadero origen biológico y su procedencia; así como los derechos fundamentales de la madre biológica, visto que esta práctica tiene un efecto negativo en la dignidad de la mujer en la medida en que esta sea considerada como un objeto que forma parte de un contrato, y no como un individuo de valor.

Frente a ello, se cree necesario que las autoridades y organismos jurídicos reconozcan la importancia de efectuar un análisis detallado de la realidad que se presenta dentro de cada nación con el propósito de implantar una normatividad orientada a velar por el desarrollo pertinente de la maternidad subrogada y así poder garantizar el respeto de las partes involucradas, principalmente del menor al aplicar en todo momento el principio del interés superior del niño.

\section{REFERENCIAS BIBLIOGRAFICAS}

Ales, M. (2020). La dignidad humana y el derecho de disposición sobre el propio cuerpo. Reflexiones a partir del rechazo de tratamientos médicos y los acuerdos de maternidad subrogada. Díkaion, 29(1), 39-65. https://doi.org/10.5294/dika.2020.29.1.2

Arbaiza, L. (2019). Cómo elaborar una tesis de grado. AlfaOmega ESAN

Arroyo, A. (2020). Gestación por sustitución: La dignidad humana en juego. Estudios de Deusto, 68(2), 41-73. https://doi.org/10.18543/ed-68(2)-2020pp41-73

Ávila, C. (2017). La maternidad subrogada en el Derecho Comparado. Cadernos de Dereito Actual, 313-344. http://www.cadernosdedereitoactual.es/ojs/index.php/cadernos/article/view/101/124 Barahona-Cobos, D. y Guerra-Coronel, M. (2021). La maternidad subrogada en el Ecuador. Revista Científica Fomento de la investigación y publicación en Ciencias Administrativas, $\quad$ Económicas $y \quad$ Contables, 6(1), 191-214. https://doi.org/10.23857/fipcaec.v6i1.337 
Castellanos, J. (2019). Análisis de la maternidad subrogada como nueva tecnología en el ámbito biomédico y jurídico-filosófico. Revista sobre la infancia y la adolescencia, (17), 62-80. https://dialnet.unirioja.es/servlet/articulo?codigo=7115727

Cossutta, C. (2018). Maternal relations, feminism and surrogate motherhood in the Italian context. Modern Italy, 23(2), 215-226. https://doi.org/10.1017/mit.2018.7

Emaldi, A. (2018), La maternidad subrogada vulnera el principio constitucional de la seguridad jurídica. La imperiosa necesidad de buscar una solución al problema español: cambio legislativo o cumplimiento de la ley. Dilemata, (28), 123-135. https://dialnet.unirioja.es/servlet/articulo?codigo $=6694798$

Fernández, P. (2018). Gestación subrogada, ¿Cuestión de derechos?. Dilemata, (26), 2737.

Fiestas, R. y Chanduví, V. (2017). La maternidad subrogada y la impugnación judicial de paternidad en el Perú. Pueblo Continente, 28(1), 201-206. http://200.62.226.189/PuebloContinente/article/view/768

Garibo, A. (2017). El interés superior del menor en los supuestos de maternidad subrogada. Cuadernos de Bioética, 28(2), 245-259. https://www.redalyc.org/pdf/875/87551223008.pdf

Gonzáles, A. (2019). Derechos fundamentales: Y subrogación materna en México: la regulación en tabasco Sinaloa y ciudad de México. UACJ Revista Especializada en Investigación Jurídica, 4(7), 167-187. http://dx.doi.org/10.20983/reij.2020.2.8

Lamm, E. (2016). Una vez más sobre gestación por sustitución, porque sin marco legal se siguen sumando violaciones a derechos humanos. Ars Iuris Salmanticensis, 4, 61107. https://revistas.usal.es/index.php/ais/article/view/14368

Lázaro, C. (2019). El concepto de persona como elemento clave de la identidad europea: El caso de la maternidad subrogada. Cuadernos Europeos De Deusto, (2), 189-201. https://ced.revistas.deusto.es/article/view/1555

Martínez, H. (2018), Maternidad subrogada. Revista Venezolana de Legislación y Jurisprudencia, (10), 269-284. http://rvlj.com.ve/wpcontent/uploads/2018/07/Revista-No.-10-1-269-284.pdf

Matia, F. (2019). ¿Resulta oportuno dar un tratamiento jurídico a la gestación subrogada en nuestro país?. Revista de Derecho Político, 1(105), 81-125. http://revistas.uned.es/index.php/derechopolitico/article/view/25269 
Múrtula, V. (2020). El bienestar del menor por nacer: ¿un interés a ponderar en el acceso a las técnicas de reproducción asistida?. Actualidad Jurídica Iberoamericana, (13), 930-959.

http://www.revista-aji.com/wpcontent/uploads/2020/09/31._Virginia_M\%C3\%83\%C2\%BArtula_pp._930959.pdf

Nuñez, A. (2015). Derechos reproductivos de la mujer infértil en el Perú: acceso a la técnica de reproducción asistida de maternidad subrogada. Foro Jurídico, (14), 8999. http://revistas.pucp.edu.pe/index.php/forojuridico/article/view/13753

Ortega-Carbajal, M., Hernández-Mosqueda, J. y Tobón-Tobón, S. (2015). Análisis documental de la gestión del conocimiento mediante la cartografía conceptual. $R a$ Ximhai, 11(4), 141-160. Recuperado de: https://www.redalyc.org/pdf/461/46142596009.pdf

Posadas, R. (2018). El derecho a la identidad y el Registro Nacional de Cedentes de gametos y embriones. Persona y Familia: Revista del Instituto de la Familia, 1(6), 123-144. https://doi.org/10.33539/peryfa.2017.n6.473

Regalado, M. (2016). Efectos, consecuencias y regulación de la maternidad subrogada. $\begin{array}{llll}\text { Femeris, } & 2(2), & 10-34 . & \text { https://e- }\end{array}$ revistas.uc3m.es/index.php/FEMERIS/article/download/3756/2356

Romero, C. (2018). Maternidad subrogada: lagunas en el ordenamiento jurídico colombiano. ¿Con qué elementos cuenta el juez para adoptar su decisión?. Revista IUSTA,

175-189. https://revistas.usantotomas.edu.co/index.php/iusta/article/view/4891/4606

Rupay, L. (2018). La maternidad subrogada gestacional altruista en el Perú: problemática y desafíos actuales. Derecho \& Sociedad, (51), 103-117. http://revistas.pucp.edu.pe/index.php/derechoysociedad/article/view/20862

Sarasol, C. y Ramón, F. (2021). La gestión subrogada: Aspectos éticos y jurídicos en el derecho español. Jurídicas $\quad$ 323-366. https://doi.org/10.17981/juridcuc.17.1.2021.12

Torres, G., Shapiro, A. \& Mackey, T. (2019). A review of surrogate motherhood regulation in south American countries: pointing to a need for an international legal framework. BMC Pregnancy and Childbirth, 19(46), 1-12. https://doi.org/10.1186/s12884-019-2182-1 
Valero, A. (2019). La maternidad subrogada: Un asunto de derechos fundamentales. $\begin{array}{lllll}\text { Teoría } & y & \text { Realidad } & \text { Constitucional, } & \text { 1(43), }\end{array}$ https://doi.org/10.5944/trc.43.2019.24433

Vasilieva, V., Stefanyshyn, N. \& Skhab-Buchynska, T. (2018). Surrogate Motherhood: Analysis of the Basis of the Legislation of Ukraine and Foreign Countries. Journal of Advanced Research in Law and Economics, 9(6), 2176-2189. https://doi.org/10.14505//jarle.v9.6(36).33

Ventura-León, J. y Barboza-Palomino, M. (2017). El tamaño de la muestra: ¿Cuántos participantes son necesarios en estudios cualitativos?. Revista Cubana de Información en Ciencias de la Salud, 28(3). 1-2. http://scielo.sld.cu/scielo.php?script=sci_arttext\&pid=S2307-21132017000300009

Vilar, S. (2019). La inseguridad jurídica derivada de la insuficiente regulación de la gestación subrogada en España. Cuadernos de Derecho Transnacional, 11(2), 815823. https://e-revistas.uc3m.es/index.php/CDT/article/view/5023/3486 